ANNALES

POLONICI MATHEMATICI

$97.3(2010)$

\title{
Homology of representable sets
}

\author{
by Marian Mrozek and Bogdan Batko (Nowy Sącz and Kraków)
}

\begin{abstract}
We generalize the notion of cubical homology to the class of locally compact representable sets in order to propose a new convenient method of reducing the complexity of a set while computing its homology.
\end{abstract}

1. Introduction. In this note we present a new reduction method for preprocessing homology computations. The method is based on the notion of the homology of representable sets modelled on the single space homology theory (see [6, 11]). In this theory there is no need for relative homology to build the long exact sequence. We make use of this in a purely combinatorial and self-contained setting. Such an approach enables a deeper reduction than in the classical case of cubical homology.

The need for very efficient homology algorithms arises from many applications, in particular to rigorous numerics of dynamical systems, where one can easily encounter cubical sets consisting of millions of cubes. The existing methods are not good enough to find homology of such sets in reasonable time. The classical algorithm for homology computations is based on Smith diagonalization of the matrix of the boundary homomorphism [13, Section 1.11]. The computational complexity of the best available Smith diagonalization algorithm is $O\left(n^{3.376 \cdots}\right)$ ([14]). Various alternatives for the classical approach have also been developed [7, 1, 5, 3, 15. Delfinado and Edelsbrunner [2] present an algorithm for Betti numbers which runs in near linear time, but the applicability of this algorithm is restricted to dimension three. Some improvements in the Smith algorithm may be obtained by applying probabilistic methods [3, 4]. Unfortunately, such methods cannot be used in rigorous numerics.

Smith diagonalization is a purely algebraic method. To speed up algorithmic homology computations one may consider methods specific to computational topology. The methods of chain complex reduction originated in

2010 Mathematics Subject Classification: Primary 55-04, 55N35; Secondary 52B99. Key words and phrases: homology algorithm, representable set, reduction method. 
[9] and then developed in [10, 8, 12, constitute such an approach. They consist in iterating the process of replacing the chain complex by a smaller one with the same homology and computing the homology only when no more reductions are possible. This way one postpones the process of computing the homology of the chain complex until the complex is acceptably small.

In this paper we show how one can benefit from the elementary reductions in homology computations by extending the cubical homology introduced in [8] to locally compact representable sets. This larger class of sets enables significantly deeper reductions than in the realm of cubical homology. This results in much faster homology algorithms for cubical sets.

This paper concentrates on the theoretical foundations of the new method. Details of the algorithm, numerical experiments and generalizations are in progress and will be published elsewhere.

2. Homology of representable sets. In what follows, essential use will be made of the notion of cubical homology introduced in [8].

If $I$ is an interval then the associated elementary cell is (cf. [8, Definition 2.13])

$$
\stackrel{\circ}{I}= \begin{cases}(l, l+1) & \text { if } I=[l, l+1], \\ {[l]} & \text { if } I=[l, l] .\end{cases}
$$

For a general elementary cube $Q=I_{1} \times \cdots \times I_{d} \subset \mathbb{R}^{d}$ we define the associated elementary cell as

$$
\stackrel{\circ}{Q}=\stackrel{\circ}{I}_{1} \times \cdots \times{\stackrel{\circ}{I_{d}}} .
$$

We let the dimension of $Q$ be the number of nondegenerate components in $Q$. Elementary cells in $\mathbb{R}^{2}$ are shown in Figure 1.
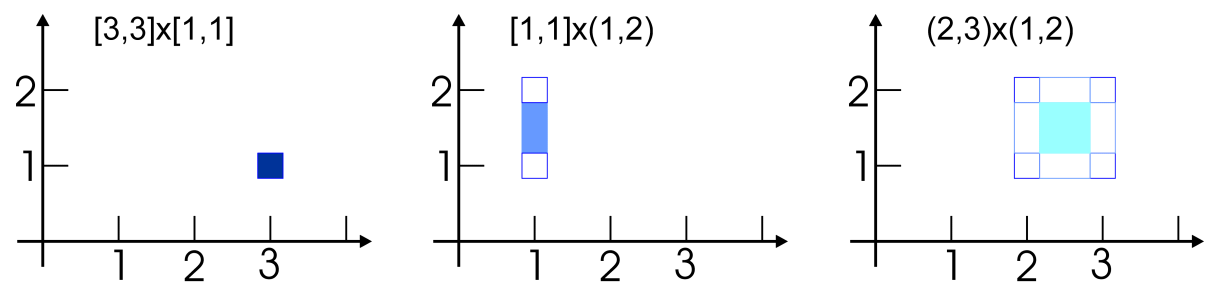

Fig. 1. Cells in $\mathbb{R}^{2}$ : 0-dimensional, 1-dimensional and 2-dimensional

Definition 1 (cf. [8, Definition 6.1]). A set $X \subset \mathbb{R}^{d}$ will be called representable if it is a finite union of elementary cells (see Figure 2).

We denote the set of all $k$-dimensional cells of $X$ by

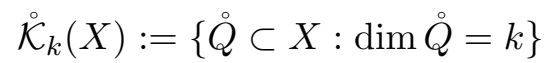




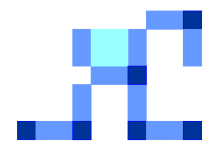

Fig. 2. A representable set in $\mathbb{R}^{2}$

and the set of all cells of $X$ by

$$
\stackrel{\mathcal{K}}{ }(X):=\bigcup_{k=0}^{d} \dot{\mathcal{K}}_{k}(X) .
$$

It is obvious that every cubical set is representable but the converse is not true.

We are going to endow a representable set $X$ with an algebraic structure identifying any elementary cell $\stackrel{Q}{Q} \in \mathcal{K}(X)$ with an algebraic object $\hat{Q}$. Let $\hat{\mathcal{K}}(X):=\{\hat{Q}: \stackrel{Q}{Q} \in \mathcal{K}(X)\}$. A finite sum

$$
c=\sum_{i=1}^{m} \alpha_{i} \hat{Q}_{i} \quad \text { with } \hat{Q}_{i} \in \hat{\mathcal{K}}_{k}(X), \alpha_{i} \in \mathbb{Z}
$$

will be called a $k$-chain of the representable set $X$. We will denote by $C_{k}(X)$ the set of all $k$-chains of $X$. Observe that $C_{k}(X)$ is a free abelian group generated by $\hat{\mathcal{K}}_{k}(X)$, with respect to the ordinary chain addition as group operation. Going towards the homology we connect the algebra and the topology of $X$ by defining the boundary of a chain. Observe that at least on the level of algebra it does not matter if a given chain is generated by elementary cells or elementary cubes. Thus, one may treat any chain of a representable set as a cubical one. Keeping this in mind we define the boundary $\stackrel{\partial}{\partial}_{k}^{X}(c)$ of a chain $c \in C_{k}(X)$ as the cubical boundary $\partial_{k}(c)$ projected onto $\dot{\mathcal{K}}_{k-1}(X)$. For instance, if $X=(1,2]$ we let $\partial_{1}^{X}(\widehat{(1,2)})=\widehat{[2]}$.

Definition 2. Given $k \in \mathbb{Z}$, we define the cell boundary operator

by

$$
\stackrel{\partial}{k}_{k}^{X}: C_{k}(X) \rightarrow C_{k-1}(X)
$$

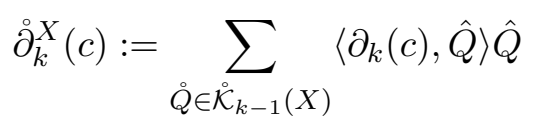

where $\partial_{k}$ stands for the ordinary cubical boundary operator and $\langle\cdot, \cdot\rangle$ denotes the scalar product of chains, i.e. $\left\langle c_{1}, c_{2}\right\rangle:=\sum_{i=1}^{m} \alpha_{i} \beta_{i}$ for $c_{1}=\sum_{i=1}^{m} \alpha_{i} \hat{Q}_{i}$ and $c_{2}=\sum_{i=1}^{m} \beta_{i} \hat{Q}_{i}$.

If $X$ is a cubical set then $\partial^{X} \equiv \partial^{X}$. Therefore we simplify the notation in the sequel and omit "o". Similarly, if the set $X$ is clear from context we will write $\partial$ instead of $\partial^{X}$. 
The linearity of both the cubical boundary operator and the scalar product of chains implies

Proposition 1. $\partial_{k}: C_{k}(X) \rightarrow C_{k-1}(X)$ is linear.

One of the most required properties of the boundary operator is $\partial \circ \partial \equiv 0$. Observe that in general this is not true.

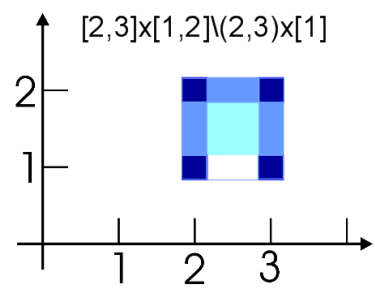

Fig. 3. $X=[2,3] \times[1,2] \backslash(2,3) \times[1]$

EXAmple 1. Consider the representable set $X=[2,3] \times[1,2] \backslash(2,3) \times[1]$. Then

$$
\partial(\partial((2, \widehat{3) \times(1}, 2)))=[\widehat{2] \times[1}]-[\widehat{3] \times[1} .
$$

We write $Q \prec R$ if a cube $Q$ is a proper face of a cube $R$, i.e. $Q \subset R$ and $Q \neq R$.

Lemma 1. A representable set $X \subset \mathbb{R}^{d}$ is locally compact if and only if for arbitrary cubes $Q \prec R \prec S$ with $\stackrel{\circ}{Q}, \stackrel{\circ}{S} \in \mathcal{K}(X)$ we have $\stackrel{R}{R} \in \mathcal{K}(X)$.

Proof. Assume that $X$ is locally compact and consider arbitrary cubes $Q \prec R \prec S$ with $\stackrel{\circ}{Q}, \stackrel{\circ}{S} \in \mathcal{K}(X)$. We need to prove that $\stackrel{\circ}{R} \in \mathcal{K}(X)$. Suppose the contrary and consider $z \in \dot{Q}$. There exists a sequence $\left(z_{n}\right)_{n \in \mathbb{N}}$ in $\stackrel{\circ}{R}$ such

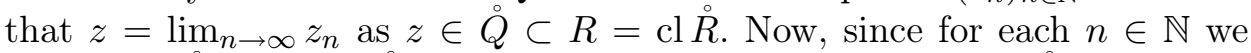
have $z_{n} \in \stackrel{\circ}{R} \subset S=\operatorname{cl} \stackrel{\circ}{S}$, there exists a sequence $\left(z_{n_{m}}\right)_{m \in \mathbb{N}}$ in $\stackrel{\circ}{S}$ convergent to $z_{n}$. Thus, in an arbitrary neighborhood of $z \in X$ there exists a limit of points in $X$ which does not belong to $X$. This means that $X$ is not locally compact and brings a contradiction.

To prove the converse assume that for arbitrary cubes $Q \prec R \prec S$ with $\stackrel{\circ}{Q}, \stackrel{\circ}{S} \in \mathcal{K}(X)$ we have $\stackrel{\circ}{R} \in \mathcal{K}(X)$. If $\operatorname{cl} X \backslash X$ is closed then $X$ is locally compact as a difference of compact sets. Thus, assume the contrary and consider $\stackrel{\circ}{R} \subset \operatorname{cl} X \backslash X$ with $R \cap X \neq \emptyset$. One can find $Q \subset R$ such that

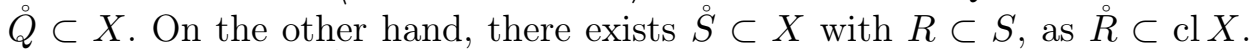
But this results in $\stackrel{\circ}{R} \subset X$, which contradicts our assumption.

TheOREM 1. If $X \subset \mathbb{R}^{d}$ is representable and locally compact then $\partial \circ \partial \equiv 0$. 
Proof. Since $\partial$ is linear, it suffices to prove that $\partial_{k-1}\left(\partial_{k}(\hat{S})\right)=0$ for any given elementary cell $\stackrel{\stackrel{S}{S} \in \mathcal{K}}{\mathcal{K}}(X), k \in \mathbb{Z}$. Let us observe that

$$
\begin{aligned}
\partial_{k-1}^{S}\left(\partial_{k}^{S}(\hat{S})\right) & =\partial_{k-1}^{S}\left(\sum_{R \in \mathcal{K}_{k-1}(S)}\left\langle\partial_{k}^{S} \hat{S}, \hat{R}\right\rangle \hat{R}\right) \\
& =\sum_{R \in \mathcal{K}_{k-1}(S)}\left\langle\partial_{k}^{S} \hat{S}, \hat{R}\right\rangle \sum_{Q \in \mathcal{K}_{k-2}(S)}\left\langle\partial_{k-1}^{S} \hat{R}, \hat{Q}\right\rangle \hat{Q} \\
& =\sum_{Q \in \mathcal{K}_{k-2}(S)}\left(\sum_{R \in \mathcal{K}_{k-1}(S)}\left\langle\partial_{k}^{S} \hat{S}, \hat{R}\right\rangle\left\langle\partial_{k-1}^{S} \hat{R}, \hat{Q}\right\rangle\right) \hat{Q} \\
& =\sum_{Q \in \mathcal{K}_{k-2}(S)}\left(\sum_{R \in \mathcal{K}_{k-1}(S): Q \prec R \prec S}\left\langle\partial_{k}^{S} \hat{S}, \hat{R}\right\rangle\left\langle\partial_{k-1}^{S} \hat{R}, \hat{Q}\right\rangle\right) \hat{Q} .
\end{aligned}
$$

This means that for each elementary cube $Q$ with $\operatorname{dim} Q=k-2$ we have

$$
\sum_{R \in \mathcal{K}_{k-1}(S): Q \prec R \prec S}\left\langle\partial_{k}^{S} \hat{S}, \hat{R}\right\rangle\left\langle\partial_{k-1}^{S} \hat{R}, \hat{Q}\right\rangle=0
$$

as $\partial^{S} \circ \partial^{S} \equiv 0$ in a cubical set $S$. On the other hand, proceeding in a similar way with respect to $\partial^{X}$ we have

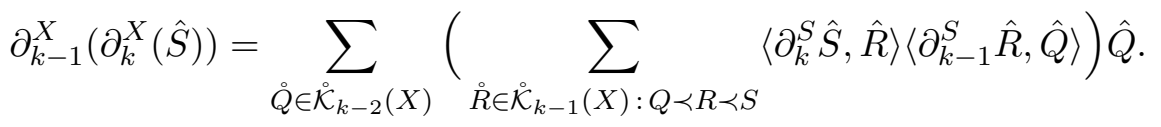

Now it is enough to show that

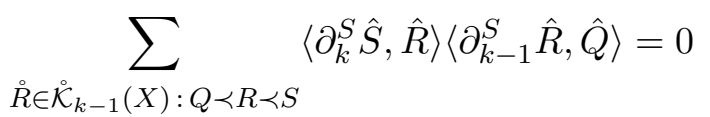

for each $\stackrel{\circ}{Q} \dot{\mathcal{K}}_{k-2}(X)$. Assume the contrary. Then comparing (2) and (3) we

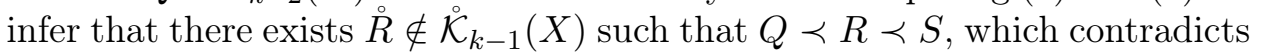
the local compactness of $X$ and completes the proof.

Now we are in a position to define the homology of $X$.

Definition 3. Let $X \subset \mathbb{R}^{d}$ be representable and locally compact. The $k$ th cell homology group of $X$ is the quotient group

$$
H_{k}(X):=Z_{k}(X) / B_{k}(X),
$$

where $Z_{k}(X):=\operatorname{ker} \partial_{k}$ is the set of all $k$-cycles of $X$ and $B_{k}(X):=\operatorname{im} \partial_{k+1}$ is the set of all $k$-boundaries of $X$. Observe that both $Z_{k}(X)$ and $B_{k}(X)$ are subgroups of $C_{k}(X)$. The sequence of all homology groups of $X$ is called the homology of $X$ and is denoted by

$$
H(X):=\left\{H_{k}(X)\right\}_{k \in \mathbb{Z}} .
$$




\section{Exact sequence}

Theorem 2. If $X, A \subset \mathbb{R}^{d}$ are representable and locally compact and $A$ is closed in $X$ then $X \backslash A$ is representable and locally compact. Moreover, there exists a long exact sequence

$$
\cdots \rightarrow H_{k}(A) \rightarrow H_{k}(X) \rightarrow H_{k}(X \backslash A) \rightarrow H_{k-1}(A) \rightarrow \cdots .
$$

Proof. Under the assumptions of the theorem, $X \backslash A$ is locally compact. By Proposition 6.3 in [8] it is representable. Consider the sequence

$$
0 \rightarrow C(A) \stackrel{i}{\rightarrow} C(X) \stackrel{j}{\rightarrow} C(X \backslash A) \rightarrow 0,
$$

where $i: C(A) \rightarrow C(X)$ is inclusion and $j: C(X) \rightarrow C(X \backslash A)$ is the linear extension of

$$
j(\hat{Q}):= \begin{cases}\hat{Q} & \text { if } \hat{Q} \in \hat{\mathcal{K}}(X \backslash A), \\ 0 & \text { if } \hat{Q} \in \hat{\mathcal{K}}(A) .\end{cases}
$$

Since $X, A$ and $X \backslash A$ are representable and locally compact, $\left\{C(A), \partial^{A}\right\}$, $\{C(X), \partial\}$ and $\left\{C(X \backslash A), \partial^{X \backslash A}\right\}$ are chain complexes. It is easy to prove that $i$ is a chain map and the sequence (5) is exact. We will show that $j$ is a chain map. Indeed, if $\hat{Q} \in \hat{\mathcal{K}}(A)$ then $\partial_{k}^{X \backslash A}\left(j_{k}(\hat{Q})\right)=\partial_{k}(0)=0$. On the other hand, since $A$ is closed we have $\partial_{k}(\hat{Q})=\sum_{P \prec Q} \alpha_{P} \hat{P}$ for suitable $\alpha_{P}$ and $j_{k-1}\left(\partial_{k}(\hat{Q})\right)=j_{k-1}\left(\sum_{P \prec Q} \alpha_{P} \hat{P}\right)=\sum_{P \prec Q} \alpha_{P} j_{k-1}(\hat{P})=0$. Now let $\hat{Q} \in \hat{\mathcal{K}}(X \backslash A)$. We have

$$
\partial_{k}\left(j_{k}(\hat{Q})\right)=\partial_{k}(\hat{Q})=\sum_{\stackrel{\rho}{P} \in \dot{\mathcal{K}}_{k-1}(X \backslash A)}\left\langle\partial_{k}^{Q}(\hat{Q}), \hat{P}\right\rangle \hat{P}
$$

and

$$
\begin{aligned}
j_{k-1}\left(\partial_{k}(\hat{Q})\right) & =j_{k-1}\left(\sum_{\dot{P} \in \dot{\mathcal{K}}_{k-1}(X)}\left\langle\partial_{k}^{Q}(\hat{Q}), \hat{P}\right\rangle \hat{P}\right) \\
& =\sum_{\dot{P} \in \dot{\mathcal{K}}_{k-1}(X)}\left\langle\partial_{k}^{Q}(\hat{Q}), \hat{P}\right\rangle j_{k-1}(\hat{P})=\sum_{\stackrel{\dot{P}^{\prime}}{\mathcal{K}_{k-1}(X \backslash A)}}\left\langle\partial_{k}^{Q}(\hat{Q}), \hat{P}\right\rangle \hat{P} .
\end{aligned}
$$

Now the exactness of (4) follows from basic homological algebra.

4. Relative homology. As we saw in Section 3 there is no need of relative homology in building the exact sequence. In this section we show how the homology of representable sets may be compared with relative homology.

Let $X, A \subset X$ be a pair of representable and locally compact sets. Associated to these sets are the groups $C(X)$ and $C(A)$ with bases $\hat{\mathcal{K}}(X)$ and $\hat{\mathcal{K}}(A)$, respectively. Since $\hat{\mathcal{K}}(A) \subset \hat{\mathcal{K}}(X)$, the quotient group $C_{k}(X) / C_{k}(A)$ is a free abelian group. Thus we have the following definitions. 
Definition 4. Let $X$ and $A \subset X$ be representable and locally compact sets. The relative chains of $X$ modulo $A$ are the elements of the free abelian groups

$$
C_{k}(X, A):=C_{k}(X) / C_{k}(A) \text {. }
$$

The relative chain complex of $X$ modulo $A$ is given by

$$
\left\{C_{k}(X, A), \partial_{k}^{(X, A)}\right\}
$$

where $\partial_{k}^{(X, A)}: C_{k}(X, A) \rightarrow C_{k-1}(X, A)$ is the boundary map induced by the standard boundary map on $C_{k}(X)$. One can verify that the induced boundary map is well defined and $\partial_{k-1}^{(X, A)} \partial_{k}^{(X, A)}=0$. We define the relative $k$-cycles

$$
Z_{k}(X, A):=\operatorname{ker} \partial_{k}^{(X, A)}
$$

the relative $k$-boundaries

$$
B_{k}(X, A):=\operatorname{im} \partial_{k+1}^{(X, A)},
$$

and finally the relative homology groups

$$
H_{k}(X, A):=Z_{k}(X, A) / B_{k}(X, A) .
$$

We now prove that the cell homology is actually the relative homology.

TheOREM 3. Let $X, A \subset X$ be a pair of representable and locally compact sets such that $X \backslash A$ is locally compact. Then

$$
H(X, A) \cong H(X \backslash A) .
$$

Proof. Consider the map $f_{k}: C_{k}(X, A) \rightarrow C_{k}(X \backslash A)$ given by

$$
f_{k}([c]):=\sum_{Q \in \mathcal{K}_{k}(X \backslash A)}\langle c, \hat{Q}\rangle \hat{Q} \quad \text { for }[c] \in C_{k}(X, A) .
$$

One can show that $f$ is well defined and is an isomorphism. Moreover,

$$
f_{k-1} \partial_{k}^{(X, A)}=\partial^{(X \backslash A)} f_{k},
$$

thus $f$ is a chain isomorphism, so it induces an isomorphism in homology.

Observe that if a set $X$ is representable and locally compact then $\operatorname{cl} X \backslash X$ is locally compact and, by Proposition 6.3 in [8], representable. Thus, as an immediate consequence of Theorem 3 we have the following corollary.

COROLlary 1. If $X$ is a representable and locally compact set then $H(X) \cong H(\operatorname{cl} X, \operatorname{cl} X \backslash X)$.

\section{Reduction method}

Definition 5. A representable set $A$ will be called a 0 -space if $H_{k}(A)$ $=0$ for each $k \in \mathbb{Z}$.

The existence of a long exact sequence results in the following theorem. 
TheOREM 4. Let $X \subset \mathbb{R}^{d}$ be a representable and locally compact set and let $A \subset X$ be a 0 -space. If $A$ is either closed or open in $X$ then

$$
H(X) \cong H(X \backslash A) \text {. }
$$

Proof. If $A$ is closed in $X$ then by Theorem 2 the sequence

$$
\cdots \rightarrow 0 \rightarrow H_{k}(X) \stackrel{\varphi_{*}}{\rightarrow} H_{k}(X \backslash A) \rightarrow 0 \rightarrow \cdots
$$

is exact. Hence $\varphi_{*}: H_{k}(X) \rightarrow H_{k}(X \backslash A)$ is an isomorphism. If $A$ is open in $X$ then one can apply Theorem 2 with respect to $X \backslash A$ and proceed as above to finish the proof.

The above theorem provides us with a convenient method to reduce the complexity of a set while computing its homology.
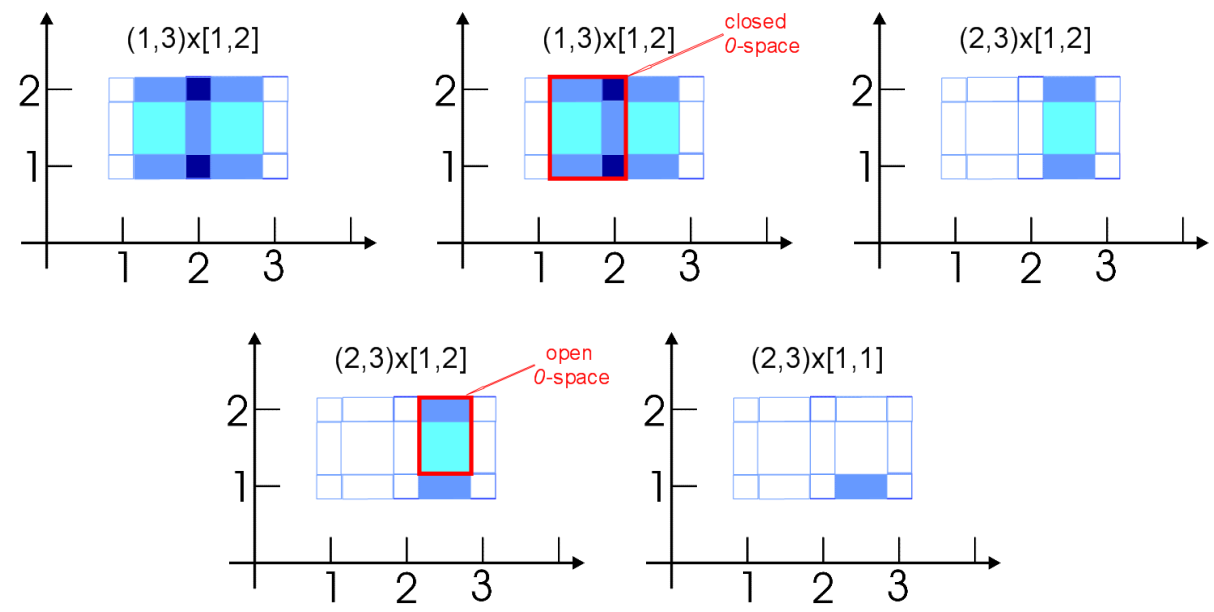

Fig. 4. Reduction via 0 -spaces

EXAmple 2. Let us find the homology of $X=(1,3) \times[1,2]$. Step by step we eliminate closed or open 0 -spaces (see Figure 4 ). Finally, we get

$$
H_{k}(X) \cong \begin{cases}\mathbb{Z} & \text { for } k=1, \\ 0 & \text { for } k \neq 1 .\end{cases}
$$

As was mentioned in the introduction, our approach enables a deeper reduction than in the classical case of cubical homology, which results in much faster homology algorithms for cubical sets. In order to visualize this, consider the cubical set $\Gamma^{1}$ shown in Figure 5. First observe that no classical reduction is possible as each vertex belongs to the boundary of exactly two edges, so there are no free faces. However, by the exact sequence (4) one can see that, for any vertex $v$ of $\Gamma^{1}$, we have $H_{k}\left(\Gamma^{1}\right)=H_{k}\left(\Gamma^{1} \backslash v\right)$ for $k \neq 0$ and the difference between the homology groups in dimension 0 is $\mathbb{Z}$. Thus in 


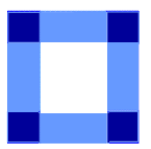

Fig. 5. $\quad \Gamma^{1}$

order to find $H\left(\Gamma^{1}\right)$ it is enough to compute the homology of the set $\Gamma^{1} \backslash v$ which admits reductions. The resulting sequence of reductions is presented in Figure 6. Now the outcome is a complex consisting of exactly one edge whose

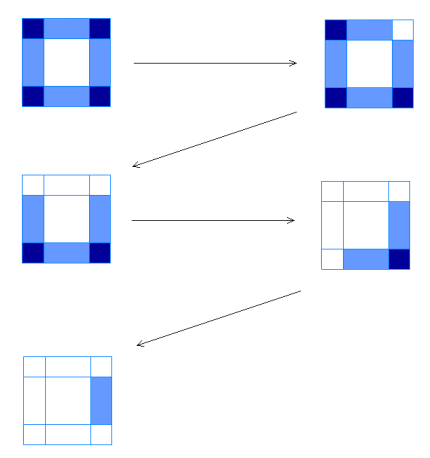

Fig. 6. Reduction of $\Gamma^{1}$ via 0 -spaces

boundary is zero. Therefore this generator is also a homology generator.

Acknowledgements. This research was partially supported by Polish MNSzW, Grant N201 037 31/3151.

\section{References}

[1] S. Basu, On bounding the Betti numbers and computing the Euler characteristic of semi-algebraic sets, Discrete Comput. Geom. 22 (1999), 1-18.

[2] C. J. A. Delfinado and H. Edelsbrunner, An incremental algorithm for Betti numbers of simplicial complexes on the 3-sphere, Computer Aided Geom. Design 12 (1995), 771-784.

[3] J.-G. Dumas, F. Heckenbach, D. Saunders and V. Welker, Computing simplicial homology based on efficient Smith normal form algorithms, in: Algebra, Geometry and Software Systems, Springer, 2003, 177-207.

[4] J.-G. Dumas, B. D. Saunders and G. Villard, On efficient sparse integer matrix Smith normal form computations, J. Symbolic Comput. 32 (2001), 71-99.

[5] H. Edelsbrunner, D. Letscher and A. Zomorodian, Topological persistence and simplification, Discrete Comput. Geom. 28 (2002), 511-533.

[6] S. Eilenberg and N. Steenrod, Foundations of Algebraic Topology, Princeton Univ. Press, 1952. 
[7] J. Friedman, Computing Betti numbers via combinatorial Laplacians, Algorithmica 21 (1998), 331-346.

[8] T. Kaczynski, K. Mischaikow and M. Mrozek, Computational Homology, Appl. Math. Sci. 157, Springer, New York, 2004.

[9] T. Kaczynski, M. Mrozek and M. Ślusarek, Homology computation by reduction of chain complexes, Computers Math. Appl. 35 (1998), 59-70.

[10] W. Kalies, K. Mischaikow, and G. Watson, Cubical approximation and computation of homology, in: Conley Index Theory, Banach Center Publ. 47, Inst. Math., Polish Acad. Sci., 1999, 115-131.

[11] W. S. Massey, Homology and Cohomology Theory, Dekker, New York, 1978.

[12] K. Mischaikow, M. Mrozek and P. Pilarczyk, Graph approach to the computation of the homology of continuous maps, Found. Comput. Math. 5 (2005), 199-229.

[13] J. R. Munkres, Elements of Algebraic Topology, Addison-Wesley, 1984.

[14] A. Storjohann, Near optimal algorithms for computing Smith normal form of integer matrices, in: Proc. 1996 International Symposium on Symbolic and Algebraic Computation, ISSAC 1996, 267-274.

[15] A. Zomorodian and G. Carlsson, Computing persistent homology, Discrete Comput. Geom. 33 (2005), 249-274.

Marian Mrozek

Department of

Computational Mathematics

WSB-NLU

Zielona 27

33-300 Nowy Sącz, Poland

E-mail: Marian.Mrozek@wsb-nlu.edu.pl

and

Department of Computer Science

Jagiellonian University

Łojasiewicza 6

30-348 Kraków, Poland

E-mail: Marian.Mrozek@ii.uj.edu.pl
Bogdan Batko

Department of

Computational Mathematics

WSB-NLU

Zielona 27

33-300 Nowy Saccz, Poland

E-mail: bbatko@wsb-nlu.edu.pl and

Institute of Mathematics Pedagogical University Podchorążych 2 30-084 Kraków, Poland E-mail: bbatko@ap.krakow.pl

Received 4.12.2008

and in final form 2.8.2009 